\title{
Suborbitale Raumfahrt - Was bringt die Zukunft?
}

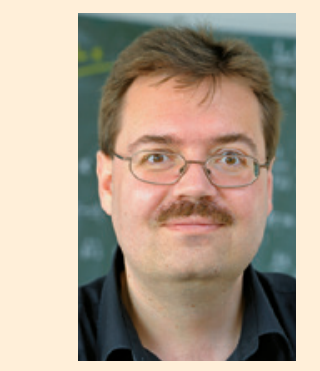

$\therefore \therefore .$.

Liebe Kolleginnen und Kollegen,

noch in den letzten Jahren war der Markt neuer kommerzieller Entwicklungen für den Suborbitalflug vielfältig, divers, unübersichtlich und mit vielen Fragezeichen behaftet. Heute ist die Situation klarer, die Entwicklungen sind deutlich weiter fortgeschritten und Planungen zur Implementierung biomedizinischer Forschung auf den neuen suborbitalen Raumfahrzeugen haben begonnen. Heute stehen zumindest 2 dieser Raumfahrzeugtypen bald vor dem Testflugbetrieb und bereits in weniger als einem Jahr könnte die Betriebsphase beginnen - neue Türen für Forschung und Entwicklung werden aufgestoßen.

Forschung auf suborbitalen Flugplattformen ist sehr anspruchsvoll und bei weitem nicht allein von dem Flugsegment abhängig: Denn Flugplattform und Bodeninfrastruktur bilden eine untrennbare Einheit. Ausgezeichnete Forschung ist ohne ausgezeichnete Bodeninfrastruktur und -logistik nicht möglich. Biowissenschaftliche Flugexperimente sind mit sehr kosten- und zeitaufwendigen Prozeduren, Standardisierungen und Testungen verbunden, eingewoben in umfassende Regelwerke und Formalitäten. Für die Konzeption, Entwicklung und Ausführung solcher Projekte ist heutzutage ein sehr hohes Maß an Spezialwissen und an wissenschaftlicher und operationeller Erfahrung erforderlich. Hier muss mit einem höchsten Grad an Sorgfalt und Redundanz geplant werden. Das Design muss so ausgelegt sein, dass das Forscherteam auch bei sich schnell oder unerwartet verändernden technischen oder operationellen Rahmenbedingungen immer handlungsfähig bleibt: Eine Synthese von kompromissloser Präzision und maximaler Flexibilität, Arbeit für Profis.

Der Erfolg des deutschen suborbitalen TEXUS-Programmes (S. 207-213) begründet sich ganz wesentlich auf die rigorose Ausrichtung an den Bedürfnissen der Wissenschaft: Biomedizinische Projekte finden auf dem Raketenstartplatz Esrange, mehr als $100 \mathrm{~km}$ nördlich des Polarkreises, eine ausgezeichnete Infrastruktur vor. Und das TEXUS-Team von Airbus DS plant und koordiniert die Forschungsmissionen zusammen mit den Wissenschaftlern und an der Wissenschaft ausgerichtet.

Die neuen suborbitalen Flugplattformen wie das Lynx-System von XCOR (S. 183-189) versprechen nicht nur eine Revolution im „Weltraumtourismus“, sondern auch in der Forschung: Experimente sollen schnell und in Sequenzen wiederholbar werden, bei deutlich kürzerer Vorbereitungszeit und massiv geringeren Kosten als in bisherigen suborbitalen Flugprogrammen. Konzept und Flugprofil sind für die Forschung tatsächlich hochinteressant und in vielen Aspekten deutlich näher an den Bedürfnissen der Wissenschaft als bisherige Flugplattformen. Für die Wissenschaft zählt am Ende aber nur das Ergebnis. Und das hängt vom Gesamtsystem ab. Die neuen suborbitalen Systeme werden noch beweisen müssen, dass sie in der Lage sind, operationelle Zuverlässigkeit mit einer wissenschaftlich modernen und zukunftsweisenden Bodeninfrastruktur zu verbinden. Wissenschaftler müssen mit dem neuen System und den gewonnenen Daten qualitativ zufrieden sein. Die Erprobung in der Wissenschaft dauert Jahre und beginnt erst ab Beginn des Flugbetriebs. Bis zu ihrer Etablierung kann durchaus ein Jahrzehnt vergehen. Dann befinden wir uns bereits in der Ära nach der Internationalen Raumstation. Hier sind neue Konzepte und Innovationen bereits heute gefragt. Zehn Jahre sind ein kurzer Atemzug in den zeitlichen Dimensionen von Forschung und Wissenschaft.

Ihr

Prof. Dr. Dr. Oliver Ullrich, Zürich / Magdeburg 